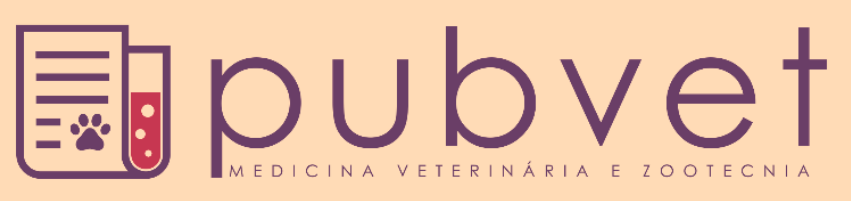

https://doi.org/10.31533/pubvet.v14n11a699.1-9

\title{
Influência da amamentação e anestro pós-parto na eficiência reprodutiva da fêmea bovina
}

\author{
Paola Risques ${ }^{1 *}$, Luiz Fernando Cozer $^{2}$, Jeniffer Claudia Silva ${ }^{1} \Phi$, Claudia Dias Monteiro Toma 9 ,

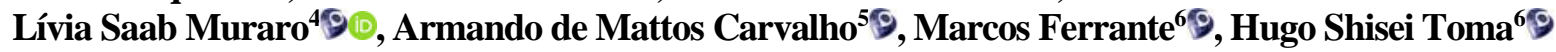

${ }^{1}$ Discente do Curso de Medicina Veterinária, Universidade de Cuiabá - UNIC, Cuiabá, MT, Brasil.

${ }^{2}$ Médico Veterinário Autônomo, Juína, MT, Brasil.

${ }^{3}$ Docente do curso de Medicina Veterinária, Centro Universitário de Lavras - UNILAVRAS, Lavras, MG, Brasil.

${ }^{4}$ Docente do curso de Medicina Veterinária, Universidade de Cuiabá - UNIC, Cuiabá, MT, Brasil.

${ }^{5}$ Docente do curso de Medicina Veterinária, Universidade Federal de Minas Gerais - UFMG, Belo Horizonte, MG, Brasil.

${ }^{6}$ Docente do curso de Medicina Veterinária, Universidade Federal de Lavras - UFLA, Lavras, MG, Brasil.

*Autor para correspondência.E-mail: paolarisques@hotmail.com

Resumo. A eficiência reprodutiva de um rebanho de cria é uma ferramenta de suma importância quando se quer aperfeiçoar a atividade pecuária de uma fazenda. Para avaliar a eficiência reprodutiva das fêmeas bovinas, algumas características devem ser levadas em consideração, como o número de concepções e bezerros nascidos em um ano, bem como o número de bezerros desmamados. Para que isso aconteça, é necessário que cada vaca consiga parir um bezerro a cada de 12 meses, e isso só será possível se o período de serviço desses animais não ultrapassar 60 a 90 dias. Este trabalho irá discutir os principais fatores que levam a um aumento no período de serviço das fêmeas e consequentemente a uma diminuição na eficiência reprodutiva. São vários os fatores que afetam a reprodução das fêmeas, dentre eles os que se destacam são: o efeito da amamentação e presença do bezerro que afeta negativamente a retomada dos ciclos estrais da fêmea, existem algumas formas de manejo de amamentação que ajudam a reduzir esse impacto negativo causado pela lactação. Como a atividade pecuária de cria requer manejos com o rebanho, esses animais sofrem um estresse causado por esse manejo, o qual gera um efeito negativo sobre a reprodução, a qual muitas vezes também é afetada pelas estações do ano e o estresse calórico. $\mathrm{O}$ anestro pós-parto e a sua fisiopatologia, também são de muita importância quando se trata de eficiência reprodutiva, assim como a sanidade e as doenças reprodutivas.

Palavras chave: baixos índices reprodutivos, eficiência reprodutiva, fêmea bovina

\section{Influence of breastfeeding and postpartum anestrus on the reproductive efficiency of the bovine female}

Abstract. The reproductive efficiency of a breeding herd is the most important tool when you want to improve the cattle industry of a farm. To evaluate the reproductive efficiency of cows, some features should be taken into consideration, as the number of conceptions and calves born in a year, and the number of calves weaned, for that to happen it is necessary that each cow can give birth to a calf every 12 months, this is only possible if the service period of these animals does not exceed 60 to 90 days. This paper will discuss the main factors leading to an increase in the length of service of females and consequently a decrease in reproductive efficiency. Several factors that affect female reproduction, including those that stand out are: the effect of breastfeeding and calf presence negatively affects resumption of estrous cycles of female, there are some forms of management of breastfeeding to help reduce this impact caused by the negative lactation. How cattle ranching requires management to create the flock, these animals suffer from stress caused 
by this management, which generates a negative effect on reproduction, which is often also affected by the seasons and heat stress. The postpartum anestrus and its pathophysiology are also very important when it comes to reproductive efficiency, as well as reproductive health and disease.

Keywords: low reproductive rates, reproductive efficiency, bovine female

\section{Influencia de la lactancia materna y el anestro posparto en la eficiencia reproductiva de la hembra bovina}

Resumen. La eficiencia reproductiva de un rebaño de cría es una herramienta
extremadamente importante cuando se trata de mejorar la actividad ganadera de una granja.
Para evaluar la eficiencia reproductiva de las hembras bovinas, se deben tener en cuenta
algunas características, como el número de terneros y terneros nacidos en un año, así como
el número de terneros destetados. Esto solo será posible si el período de servicio de estos
animales no excede de 60 a 90 días. Este trabajo discutirá los principales factores que
conducen a un aumento en el período de servicio de las hembras y, en consecuencia, a una
disminución en la eficiencia reproductiva. Existen varios factores que afectan la
reproducción de las hembras, entre los cuales se destacan los siguientes: el efecto de la
lactancia materna y la presencia del becerro afectando negativamente la reanudación de los
ciclos estrales de la hembra, existen algunas formas de manejo de la lactancia que ayudan
a reducir este impacto negativo causado por la lactancia. Como la cría de ganado requiere
manejo con el rebaño, estos animales sufren estrés causado por este manejo, que tiene un
efecto negativo en la reproducción, que a menudo también se ve afectada por las estaciones
y el estrés por calor. El anestro posparto y su fisiopatología también son muy importantes
cuando se trata de la eficiencia reproductiva, así como la sanidad y las enfermedades
reproductivas.

Palabras clave: Tasas reproductivas bajas, eficiencia reproductiva, hembras bovinas

\section{Introdução}

A atividade pecuária brasileira tem se demonstrado cada vez mais importante na economia nacional, uma vez que o Brasil é detentor de grandes áreas férteis para produção não só de carne, mas de qualquer outro tipo de produção agroindustrial, com isso, procura-se cada vez mais aumentar a eficiência produtiva e reprodutiva dos animais (Fachinetto \& Brisola, 2018).

O sistema de produção de bovinos de corte no Brasil está se desenvolvendo muito rápido, o que tem refletido no significativo aumento da taxa de desfrute nos últimos dez anos. Por outro lado, ainda estamos longe dos níveis de produtividade dos rebanhos mais competitivos do mundo (Neto, 2018; Vianna, 2007).

Para termos altos índices de produção com maior lucratividade, é preciso que ocorra perfeita interação dos parâmetros genéticos, reprodutivos, sanitários e nutricionais. Neste sentido, faz-se necessário analisarmos o desempenho reprodutivo dos animais (Santos et al., 2014). Vários são os fatores que afetam a eficiência reprodutiva nos bovinos, como fatores nutricionais, climáticos, escore de condição corporal, presença de bezerro, problemas ginecológicos, andrológicos e libido nos machos (Ferreira et al., 1997).

Os transtornos de fertilidade conduzem independentemente de sua origem, a um prolongamento do período de serviço da vaca. Todo período de serviço que se estende por mais de 90 dias, leva a prejuízos, que inclui vários componentes, como redução na produção diária de leite, menor produção de bezerros, aumento dos custos de reprodução e com mão de obra (Berchtold, 1988; Buso et al., 2018).

Para manter-se líder no mercado mundial de carne, o país ainda necessita de melhorias tecnológicas em diversos setores, de formas independentes e relacionadas, como melhoramento genético, sanidade, nutrição e reprodução dentre outros. Dessa maneira, nota-se a urgente necessidade da modificação na 
visão dos produtores rurais, adotando atitudes empresariais e de manejo capazes de aumentar os lucros através de sua produção (Neto, 2018).

Diante disso objetivou-se nesta revisão discutir sobre os principais problemas que afetam a eficiência reprodutiva das fêmeas bovinas, como também algumas estratégias para diminuir os impactos negativos de tais problemas.

\section{Endocrinologia da reprodução da fêmea bovina}

Todas as funções reprodutivas da fêmea são o resultado da interação coordenada entre sistema nervoso central, hipotálamo-hipófise, ovário e útero. O equilíbrio nas funções entre estes quatro tecidos é que irá garantir a fertilidade das fêmeas. Logo, precisamos conhecer as atividades destes componentes para que possamos interferir de forma eficiente na atividade reprodutiva da fêmea (Hafez \& Hafez, 2004).

Contudo, quando se procura melhores índices reprodutivos, é de suma importância, conhecer como ocorre o controle neuro-hormonal da reprodução, bem como sua atuação no processo reprodutivo, e o papel que cada hormônio desempenha na reprodução. É sabido que vários fatores afetam o controle neuro-hormonal, diminuindo assim a eficiência reprodutiva destes animais, com isso, é importante saber como agem esses fatores e o mecanismo pelo qual afetam as secreções hormonais, para que seja possível interferirmos nesse mecanismo, diminuindo as perdas ocasionadas por tais problemas.

\section{Efeito da amamentação na reprodução}

Inúmeras evidências comprovam a interferência da amamentação sobre o desempenho reprodutivo em vacas de leite e de corte, o que é indesejável do ponto de vista econômico e zootécnico, pois diminuem a eficiência reprodutiva de um rebanho. Os efeitos da amamentação sobre a reprodução, isolada ou associada com outro fator como a nutrição, são marcantes, pois afetam diretamente o intervalo do parto ao primeiro cio, aumenta a incidência de ovulação sem estro, dificultando a identificação do estro devido sua menor intensidade (Castro et al., 2018; Ferreira, 1992).

A amamentação é também um dos fatores que causam o anestro pós-parto em bovinos, podendo atrasar o início da atividade cíclica ovariana ou também o aparecimento de estro pós-parto. O exato mecanismo pelo qual a amamentação altera a função reprodutiva na fêmea não está ainda totalmente compreendido. Sabe-se que a sucção do leite e a presença da cria geram mensagens metabólicas neurais, sensoriais, olfativas e fisiológicas que inibem a síntese de LH ou impedem a hipófise de responder apropriadamente aos estímulos de GnRH (Buso et al., 2018; Williams, 1990).

No período imediato do pós-parto, a ausência de pulsos de LH não ocorre devido à mamada do bezerro e sim pelo esgotamento das reservas hipofisárias de LH. A mamada só afeta a retorno ao ciclo estral a partir do $20^{\circ}$ dia pós-parto, quando as reservas de LH já foram refeitas, devido à redução da onda pulsátil de LH. Isso se dá devido a inibição na liberação de GnRH ocasionado por diversos fatores, como: presença do bezerro - a estimulação da glândula mamária por si só não é capaz de exercer tal mecanismo, é necessário que a fêmea exerça algum contato com sua cria; presença de estrógenos ovarianos - a amamentação potencializa o feedback negativo do estrógeno sobre os centro de liberação de GnRH; peptídeos opioides endógenos (POE) - a amamentação associada com a presença do bezerro liberam os POE, os quais geram efeito inibitório na liberação do $\mathrm{GnRH}$ e indiretamente suprime a liberação de LH pela hipófise (Randel, 1990). O ato da mamada pode liberar glicocorticoides que agem igualmente aos POE.

Durante o período de amamentações frequentes, as concentrações de prolactina são elevadas, ao contrário das concentrações circulantes de LH e FSH, entretanto não se relaciona a inibição de LH com as concentrações de prolactina e sim à interação entre o reconhecimento da vaca com seu bezerro, e também ao ato de sucção dos tetos. A prolactina não está associada a falta de ovulação no pós-parto de vacas, pois quando utilizado bloqueador de prolactina (dopamina), não se observou mudanças nas concentrações de gonadotrofinas na duração do anestro pós-parto (Yavas \& Walton, 2000).

A diminuição nas secreções de LH faz com que os folículos ovarianos não se desenvolvam e secretam níveis baixos de estradiol por influência do FSH. O fator limitante para a baixa secreção de LH é a baixa 
liberação endógena de $\mathrm{GnRH}$, pois em administrações parenterais de $\mathrm{GnRH}$, nota-se indução na ovulação desses animais (Franco et al., 2016).

Existem algumas alternativas de manejo que podem ser utilizadas para aumentar a eficiência reprodutiva das vacas de cria em períodos de amamentação, como a mamada controlada, desmama precoce e a mamada interrompida, sendo que esses manejos tem como finalidade o aumento dos pulsos de LH, aumento das concentrações de receptores para LH e FSH nos folículos, levando a uma ovulação após poucos dias (Castro et al., 2018; Waltens et al., 1982).

\section{Mamada controlada}

O manejo da mamada controlada consiste em separar os bezerros das vacas a partir dos 30 dias de nascimento, sendo estes colocados em piquetes separados de suas mães. Os bezerros têm acesso às vacas uma ou duas vezes ao dia para mamarem, onde permanecem por cerca de meia hora e na sequência são apartados novamente. Este método embora simples apresenta uso limitado devido ao manejo intensivo, principalmente durante a primeira semana de separação, elevados custos com cercas e suplementação alimentar para os bezerros (Castro et al., 2018; Moletta et al., 2003; Oliveira et al., 2006).

Embora necessite de um manejo mais intensivo, a mamada controlada demonstra melhores resultados reprodutivos e de retorno ao estro quando comparados com o manejo tradicional, com bezerro ao pé da vaca até sua desmama com 8 meses, pois a implementação desse manejo aumentam as taxas de prenhez do rebanho, principalmente em primíparas, pois reduz o desgaste que a sucção do leite ocasiona (Bell et al., 1998).

Cubas et al. (1985) compararam as taxas de prenhez entre um rebanho de vacas submetidas a amamentação controlada e outro com sistema tradicional de amamentação, verificando resultados de $76,0 \%$ e $46,0 \%$ de taxa de prenhez, respectivamente, fato este, também observado por Pencai et al. (2011), que relataram taxa de prenhez superior para a amamentação controlada $(87,3 \%)$ em comparação ao manejo convencional, onde as vacas ficavam com os bezerros permanentemente ao pé, até 210 dias de idade $(54,5 \%)$.

\section{Desmama precoce}

O método de desmama precoce consiste na antecipação da idade a desmama, que passa a ser de 60 , 70, 90 ou 110 dias ao invés de 180 - 205 dias de idade como tradicionalmente. A antecipação da desmama ocorre principalmente devido a situações de escassez de forragens durante o período de lactação. O objetivo principal desse manejo é de permitir que as fêmeas recuperem sua condição corporal e possam conceber ainda dentro do período de monta seguinte (Castro et al., 2018; Souza Neto, 2008).

As restrições alimentares durante esse período, associado à amamentação aumenta significativamente o intervalo do parto até o primeiro estro, e como consequência, reduz os índices de prenhez do rebanho (Silva et al., 2019). Como durante a lactação as exigências nutricionais são mais elevadas do que no terço final de gestação, em casos de restrições alimentares, a atividade reprodutiva é deixada de lado com a mobilização de nutrientes voltada em sua grande maioria para a sobrevivência do bezerro (Silveira et al., 2016). Vacas primíparas são as mais prejudicadas nesses casos, devido ao fato de ainda estarem em crescimento. Contudo o manejo de desmama antecipada pode amenizar ou até solucionar esse problema, promovendo aumento nos índices de concepção e aumento a taxa de lotação das pastagens em função das vacas solteiras necessitarem de 40 a $60 \%$ menos nutrientes do que vacas com cria ao pé (Connor et al., 1990).

Segundo Arthington \& Minton (2004), o desmame precoce pode ser uma ferramenta prática e lucrativa de manejo nas propriedades de regiões tropicais. Além disso, pode diminuir em até 24 dias o período de anestro pós parto, devido ao aumento no ECC das vacas em mais 2 pontos (aprox. $70 \mathrm{~kg}$ ), resultando em incremento de aproximadamente $30 \%$ na taxa de prenhez (Silveira et al., 2016).

Quando adotado esse método, o pecuarista deve levar em consideração os gastos com a suplementação dos bezerros, uma vez que irão necessitar de alimentação sólida e de qualidade, como sendo a única fonte de nutrientes e assim garantindo bezerros de qualidade durante essa etapa (Lobato et al., 2007; Silveira et al., 2016). 
Segundo Lobato et al. (2007), o efeito do desmame precoce em vacas primíparas utilizando pastagens melhoradas apresentou menor intervalo entre partos, porém sem diferença significativa na taxa de prenhez, o desmame precoce concentrou também a parição no início da estação de partos propiciando melhores condições de recuperação das vacas para a próxima estação de monta.

Restle et al. (2001) compararam o efeito do desmame de bezerros aos três e aos sete meses de idade sobre o desempenho reprodutiva de vacas da raça Charolês e Nelore. Os resultados demonstraram que o desmame de três meses aumentou o peso, melhorou a condição corporal das vacas $(+45 \mathrm{~kg}, 3.3 \mathrm{vs}$. 2.1), aumentou a percentagem de cios e taxa de prenhez ( 81 vs. $51 \%, 67$ vs. $37 \%$ ), além de diminuir o intervalo entre parto e primeiro cio (102 vs. 114 dias) das vacas submetidas a tal manejo.

O desmame precoce de bezerros com três meses de idade ocasiona uma redução na ingestão de matéria seca, na ordem de 25 a 30\%, e por isso haveria a possibilidade de aumentar em $25 \%$ a taxa de lotação quando essas matrizes são comparadas com outras que amamentam até os 7 meses de idade do bezerro (Silva et al., 2019).

\section{Mamada interrompida "shang"}

Este método denominado mamada interrompida ou shang é o mais utilizado quando se trata de manejos de amamentação e consiste na separação temporária do bezerro, por um período variável de 48 a 72 horas, com posterior retorno junto à mãe, podendo ser utilizado a partir dos 40 dias de vida do bezerro com intervalos de $15 \mathrm{em} 15$ dias, com a finalidade de estimular o retorno da atividade ovariana (Baruselli et al., 2004; Castro et al., 2018).

Segundo Silveira \& Williams (1991), com a retirada do bezerro e sem o ato da mamada, após 24 horas de separação cessa a liberação de opioides endógenos e assim o GnRH que estava sendo inibido pela ação desses opioides, começa a ser produzido pelo hipotálamo e este, por sua vez, estimula a hipófise à produzir e liberar LH. Assim, com o aumento de LH, ocorre o restabelecimento dos ciclos estrais (Oliveira et al., 2007).

O efeito dessa separação temporária depende muito do estado nutricional da fêmea. Vacas que se encontram com baixo escore de condição corporal não respondem ao tratamento, mas quando as fêmeas estão em boas condições nutricionais, esse método pode induzir a manifestação precoce do estro no pósparto, sendo capaz de melhorar a taxa de concepção em até 30\% (Castro et al., 2018), contudo os resultados obtidos até o momento são bastante variados (Pötter, 2002). A desmama temporária ou interrompida não causa nenhum prejuízo ao peso dos bezerros à desmama definitiva (210 dias), quando comparada com o método tradicional de desmama (Castro et al., 2018).

\section{Anestro pós-parto em bovinos}

O anestro pós-parto é o período que se estende desde o parto até o aparecimento do primeiro estro, sendo caracterizado pela ausência de manifestação estral. Neste período, também conhecido como puerpério, ocorre a involução uterina e o restabelecimento da atividade ovariana. O tempo de involução ocorre por volta dos 40 dias pós-parto, e o aparecimento do cio no $50^{\circ}$ dia ocorre em $40 \%$ das vacas leiteiras e em 95\% das vacas de corte (Jainudeen \& Hafez, 2004; Ferreira et al., 2019).

$\mathrm{O}$ anestro pós-parto é o período transitório durante o qual o eixo hipotálamo-hipófise-ovário-útero se recupera de uma prévia gestação, sendo limitado por dois eventos marcantes, o parto e o aparecimento do primeiro estro. É um evento fisiológico considerado normal que ocorre logo após o parto, tornandose anormal quando excede um tempo esperado (Sartori \& Barros, 2011).

O longo período de anestro pós-parto é um dos principais fatores responsáveis pelo aumento do período de serviço e pela baixa eficiência reprodutiva do rebanho bovino (Ferreira, 1992). Segundo Yavas \& Walton (2000), o intervalo parto-concepção não deve ser superior a 75 dias, considerando que o período de gestação seja aproximadamente 290 dias. Vacas no período pós-parto apresentam anestro, e na maioria das vezes não concebem no período esperado.

Esse período depende muitas vezes de fatores ligados a fêmea e ao ambiente em que se relaciona como, idade, raça do animal, fatores genéticos, etc. A duração do anestro pós-parto é em sua grande maioria influenciada pela amamentação, estado nutricional estação de parição, entre outros, sendo que 
ocorre com maior frequência em vacas de primeira cria (Castro, 2002; Sartori \& Barros, 2011; Ferreira et al., 2019).

Na maioria das vezes, o anestro é consequência de uma série de folículos dominantes que falham em ovular devido a baixas concentrações de LH (Jolly et al., 1995; Roche et al., 1992).

Os mecanismos de controle do anestro pós-parto envolvem uma complexa relação entre hipotálamo, hipófise, ovário e útero. No pós-parto, os estoques de LH e FSH da hipófise estão reduzidos devido ao feedback negativo do estradiol $\left(\mathrm{E}_{2}\right)$ no hipotálamo e das elevadas concentrações de progesterona $\left(\mathrm{P}_{4}\right)$ no final da gestação (Mapletoft et al., 2018; Nett et al., 1988; Sartori \& Barros, 2011).

Após o parto, com a remoção da unidade feto-placentária, as concentrações circulantes de $\mathrm{E}_{2}$ e $\mathrm{P}_{4}$ decrescem dramaticamente, o que resulta na remoção do feedback negativo e permite uma retomada gradual do eixo hipotálamo-hipófise-gonadal (Nett et al., 1988). A liberação de FSH se restabelece rapidamente no período pós-parto, dentro de duas semanas pós-parto, o padrão de crescimento folicular em ondas já pode ser detectado, com a ocorrência de múltiplas ondas foliculares ocorrendo antes da primeira ovulação (Mapletoft et al., 2018; Nett et al., 1988).

Ao contrário do FSH, as concentrações de LH estão baixas no pós-parto imediato e o retorno ao padrão secretório compatível com a ciclicidade ocorre de maneira gradual nos primeiros 30 dias pósparto. Entre os dias 15 e 30 pós-parto a responsividade do hipotálamo ao $E_{2}$ é restabelecida (Nett et al., 1988).

O primeiro folículo dominante pode desenvolver-se completamente e ovular ou tornar-se atrésico e ser substituído por um ou mais folículos dominantes subsequentes ou ainda continuar seu crescimento e tornar-se cístico (Sartori \& Barros, 2011; Wiltbank et al., 2002).

\section{Relações entre sanidade e eficiência reprodutiva em bovinos}

O manejo sanitário dos bovinos de corte exerce importância fundamental na condução dos sistemas de produção, impedindo que as enfermidades disseminem dentro do rebanho, causando prejuízos econômicos. Este compreende medidas profiláticas (vacinas, vermífugos etc.) e o controle de doenças (Castro et al., 2018). O planejamento sanitário possibilita ao produtor a redução dos riscos de produção, garantindo também um produto seguro ao consumidor no ponto final da cadeia produtiva (Andreotti et al., 1998).

Dentro de um rebanho, o interessante é realizar a prevenção das doenças, principalmente as de caráter reprodutivo. Faz-se necessário a orientação do médico veterinário, não como substituição das práticas de manejo, mas como um complemento, não só no tratamento dos animais, mas na organização e realização do controle sanitário da propriedade (Ferreira, 2010).

As principais sintomatologias de patologias da reprodução são o abortamento, retenção de placenta, bezerros nascidos fracos ou mortos, infecções uterinas, que contribuem massivamente para o aumento de intervalo entre partos, diminuindo assim a produtividade do rebanho. As principais patologias são a leptospirose, rinotraqueíte infecciosa bovina (IBR), diarreia viral bovina (BVD) e a brucelose (Baruselli et al., 2019; Castro et al., 2018; Sartori et al., 2020). Segundo Sartori et al. (2020) ocorrem uma menor perda embrionária e maior taxa de gestação em programas de IATF, com fêmeas previamente imunizadas contra IBR, BVD e leptospirose.

\section{Considerações Finais}

A eficiência reprodutiva tem extrema importância quando se busca maior lucratividade em uma fazenda. Contudo, percebe-se que os produtores rurais e os profissionais do ramo demonstram preocupação em aumentar a eficiência reprodutiva do rebanho, realizando a aplicação de métodos de amamentação, diminuindo o efeito da presença do bezerro; cuidados especiais com as primíparas e novilhas, principalmente no que diz respeito à puberdade e maturidade sexual. Treinamento de funcionários para evitar o estresse crônico nos animais, técnicas para diminuir o estresse calórico e cuidados especiais com a sanidade do rebanho. 
Com a atual realidade mundial, faz-se necessária a intensificação da produção, com o intuito de conseguir produzir cada vez mais alimentos em um menor espaço de terra, se tratando de pecuária o que se busca é a produção de mais animais por hectare ano, sendo assim, a atuação dos profissionais da Medicina Veterinária é de suma importância, pois estes colocam em prática todo seu conhecimento em prol do desenvolvimento do setor e melhores condições de vida para todos aqueles envolvidos nesse meio.

\section{Referências bibliográficas}

Andreotti, R., Gomes, A., Pires, P. P., \& Rivera, F. E. B. (1998). Planejamento sanitário de gado de corte. Embrapa Gado de Corte-Documentos (INFOTECA-E).

Arthington, J. D. \& Minton, J. E. (2004). The effect of early calf weaning on feed intake, growth, and postpartum interval in thin, Brahman-crossbred primiparous cows. The Professional Animal Scientist, 20, 1, 34-38. https://doi.org/10.15232/S1080-7446(15)31269-9

Baruselli, P.S, Reis, E. ., Marques, M. ., Nasser, L. ., \& Bó, G. . (2004). The use of hormonal treatments to improve reproductive performance of anestrous beef cattle in tropical climates. Animal Reproduction Science, 82-83, 479-486. https://doi.org/10.1016/j.anireprosci.2004.04.025

Baruselli, Pietro Sampaio, Catussi, B. L. C., Abreu, L. Â., Elliff, F. M., Silva, L. G., Batista, E. S., \& Crepaldi, G. A. (2019). Evolução e perspectivas da inseminação artificial em bovinos. Revista Brasileira de Reprodução Animal, 43(2), 308-314.

Bell, D. ., Spitzer, J. ., \& Burns, G. . (1998). Comparative effects of early weaning or once-daily suckling on occurrence of postpartum estrus in primiparous beef cows. Theriogenology, 50(5), 707-715. https://doi.org/10.1016/S0093-691X(98)00176-9

Berchtold, M. (1988). Importancia de los trastornos de fertilidad. En: Grunert, E. Y M. Berchtold. 1988. Infertilidad en la vaca. Ed. Hemisferio. Sur. Buenos Aires, Argentina.

Buso, R. R., Campos, C. C., Santos, T. R., Saut, J. P. E., \& Santos, R. M. (2018). Retained placenta and subclinical endometritis: prevalence and relation with reproductive performance of crossbred dairy cows. Pesquisa Veterinária Brasileira, 38(1), 1-5.

Castro, F. C., Fernandes, H., \& Leal, C. L. V. (2018). Sistemas de manejo para maximização da eficiência reprodutiva em bovinos de corte nos trópicos. Vet. Zoot., 41-61.

Castro, T. (2002). Anestro posparto en la vaca de cría. In Ungerfeld R. Reproducción en los animales domésticos. Montevideo, Ed. Melibea (pp. 210-217).

Connor, H. C., Houghton, P. L., Lemenager, R. P., Malven, P. V., Parfet, J. R., \& Moss, G. E. (1990). Effect of dietary energy, body condition and calf removal on pituitary gonadotropins, gonadotropinreleasing hormone $(\mathrm{GnRH})$ and hypothalamic opioids in beef cows. Domestic Animal Endocrinology, 7(3), 403-411. https://doi.org/10.1016/0739-7240(90)90045-2

Cubas, A. C., Mancio, A. B., Lesskiu, C., \& Tahira, J. K. (1985). Efeito da amamentação controlada sobre a eficiência reprodutiva de vacas de corte no sul do Paraná. Revista da Sociedade Brasileira de Zootecnia, 14, 247-255.

Fachinetto, J. D., \& Brisola, M. V. (2018). Evolução dos estudos sobre a produção de bovinos de corte e a emissão de gases de efeito estufa decorrente dessa atividade na região central do Brasil. Desenvolvimento e Meio Ambiente, 45. https://doi.org/10.5380/dma.v45i0.47354

Ferreira, A. M., Goulart, I. L. \& Almeida Neto, J. R. M. (2019). Tempo para recuperação da atividade ovariana luteal cíclica em vacas mestiças leiteiras magras com anestro. PUBVET, 13, 2, 1-7. https://doi.org/10.31533/pubvet.v13n2a269.1-7

Ferreira, A M. (2010). Reprodução da fêmea bovina: Fisiologia aplicada e problemas mais comuns (causas e tratamentos) (Vol. 1). Editar Editora.

Ferreira, A M, Sá, W. F., Viana, J. H., Fernandes, C. A., \& Camargo, L. S. (1997). Taxa de näo retorno ao cio em 60 dias e taxa de prenhez em bovinos. Arq. Bras. Med. Vet. Zootec, 261-267.

Ferreira, Ademir Moraes. (1992). Efeito da amamentação na reprodução de vacas: uma revisão. Pesquisa Agropecuária Brasileira, 27(1), 27-39.

Franco, G. L., Faria, F. J. C., \& D’Oliveira, M. C. (2016). Interação entre nutrição e reprodução em 
vacas de corte. Informe Agropecuário, 37(292), 36-53.

Hafez, E. S. E., \& Hafez, E. S. E. (2004). Anatomia da reprodução feminina. In Reprodução Animal (Vol. 6, p. 513). $7^{\text {a }}$ ed. São Paulo: Editora Manole.

Jainudeen, M. R., \& Hafez, E. S. E. (2004). Gestação, fisiologia pré-natal e parto. Reprodução Animal, 7, 141-142.

Jolly, P. D., McDougall, S., Fitzpatrick, L. A., Macmillan, K. L., \& Entwistle, K. W. (1995). Physiological effects of undernutrition on postpartum anoestrus in cows. Journal of Reproduction and Fertility, 49, 477-492. http://www.ncbi.nlm.nih.gov/pubmed/7623336

Lobato, J. F. P., Almeida, L. S. P., Osório, E. B., \& Müller, A. (2007). Efeito da idade de desmame no desenvolvimento e nas características de carcaça de novilhos de corte. Revista Brasileira de Zootecnia, 36(3), 596-602. https://doi.org/10.1590/S1516-35982007000300012

Mapletoft, R. J., Bó, G. A., Baruselli, P. S., Menchaca, A., \& Sartori, R. (2018). Evolution of knowledge on ovarian physiology and its contribution to the widespread application of reproductive biotechnologies in South American cattle. Animal Reproduction, 15(Suppl. 1), 1003-1014. https://doi.org/10.21451/1984-3143-AR2018-0007

Moletta, J. L., Perotto, D., Silva, N. L., Strack, A. G., \& Motta, J. B. O. (2003). Efeito da associação de amamentação controlada com desmame precoce no desempenho ponderal e reprodutivo de vacas de corte. Anais Da 40 Reunião Anual Da Sociedade Brasileira de Zootecnia, 53-54.

Neto, O. A. (2018). O Brasil no mercado mundial de carne bovina: análise da competitividade da produção e da logística de exportação brasileira. Ateliê Geográfico, 12(2), 183-204.

Nett, T. M., Cermak, D., Braden, T., Manns, J., \& Niswender, G. (1988). Pituitary receptors for GnRH and estradiol, and pituitary content of gonadotropins in beef cows. II. Changes during the postpartum period. Domestic Animal Endocrinology, 5(1), 81-89. https://doi.org/10.1016/0739-7240(88)90029-X

Oliveira, J. S., Zanine, A. M., \& Santos, E. M. (2007). Fisiologia, manejo e alimentação de bezerros de corte. Arquivos de Ciências Veterinárias e Zoologia da UNIPAR, 10(1), 39-48.

Oliveira, R. L., Barbosa, M. A. A. F., Ladeira, M. M., Silva, M. M. P., Ziviani, A. C., \& Bagaldo, A. R. (2006). Nutrição e manejo de bovinos de corte na fase de cria. Revista Brasileira de Saúde e Produção Animal, 7(1).

Pencai, F. W., Kozicki, L. E., Costa, C. E. M. P., Silva, N. L., Molleta, J. L., \& Motta, J. B. O. (2011). Indução ao estro pós puerperal em bovinos mestiços de corte mediante o emprego de diferentes protocolos de amamentação. Veterinária e Zootecnia, 18(1), 53-62.

Pötter, B. A. A. (2002). Manejo reprodutivo pós-parto de vacas primíparas e desenvolvimento de terneiros [Dissertação - Universidade Federal do Rio Grande do Sul. Faculdade de Agronomia. Programa de Pós-Graduação em Zootecnia.]. http://hdl.handle.net/10183/3262

Randel, R. D. (1990). Nutrition and postpartum rebreeding in catle. Journal of Animal Science, 68, 853862

Restle, J., Vaz, R. Z., Alves Filho, D. C., Bernardes, R. A. L. C., Pascoal, L. L., Senna, D. B., \& Polli, V. A. (2001). Desempenho de vacas Charolês e Nelore desterneiradas aos três ou sete meses. Revista Brasileira de Zootecnia, 30(2), 499-507. https://doi.org/10.1590/S1516-35982001000200029

Roche, J. F., Crowe, M. A., \& Boland, M. P. (1992). Postpartum anoestrus in dairy and beef cows. Animal Reproduction Science, 28(1-4), 371-378. https://doi.org/10.1016/0378-4320(92)90123-U

Santos, M. D., Junior, D. A. F., Monteiro-Toma, C. D., Toma, H. S., Freitas, S. H., Costa, D. S., \& Junior, C. K. (2014). Taxas de gestação e formação de corpo lúteo acessório em novilhas nelore tratadas com hcg após a inseminação artificial em tempo fixo. Acta Veterinaria Brasilica, 8(4), 231235. https://doi.org/10.21708/avb.2014.8.4.4236

Sartori, G. S., Perucchi, G. R. H., Pereira, D. R., \& Frias, D. F. R. (2020). Analysis of the efficiency of active immunization against IBR, BVD and leptospirosis in increasing pregnancy rate in FTAI protocols. Acta Veterinaria Brasilica, 14(2), 92-98. https://doi.org/10.21708/avb.2020.14.2.9115

Sartori, R., \& Barros, C. M. (2011). Reproductive cycles in Bos indicus cattle. Animal Reproduction Science, 124(3-4), 244-250. https://doi.org/10.1016/j.anireprosci.2011.02.006 
Short, R. E., Bellows, R. A., Staigmiller, R. B., Berardinelli, J. G., \& Custer, E. E. (1990). Physiological mechanisms controlling anestrus and infertility in postpartum beef cattle. Journal of Animal Science, 68(3), 799. https://doi.org/10.2527/1990.683799x

Silva, J. C. B., Nogueira, E., \& Nicacio, A. C. (2019). Projeto Mais Precoce Embrapa: aumentar a produção e qualidade dos bezerros. Revista Brasileira de Reprodução Animal, 43(2), 340-345.

Silveira, L. G. G., Piona, M. N. M., Mousquer, C. J., Gomes, R. S., \& Silveira, A. C. (2016). Sistemas de cria em áreas tropicais: desmama precoce. Revista Brasileira de Higiene e Sanidade Animal, 10(4), 858-869. https://doi.org/10.5935/1981-2965.20160070

Silveira, P. A., \& Williams, G. L. (1991). Effects of" own" versus" alien" suckling on luteinizing hormone secretion and interval to first ovulation in early postpartum, anestrous beef cows. Journal Animal Science, 69(Suppl I), 417.

Souza Neto, R. L. (2008). Desempenho reprodutivo de vacas de corte submetidas à prática de desmame definitivo para inseminação artificial [Mestrado em Zootecnia - Universidade Federal do Rio Grande do Sul. Faculdade de Agronomia. Programa de Pós-Graduação em Zootecnia.]. http://hdl.handle.net/10183/14978

Vianna, E. (2007). Comportamento e eficiência reprodutiva. Revista Brasileira de Reprodução Animal, Belo Horizonte, 31(2), 177-182.

Waltens, D. L., Smith, M. F., Harms, P. G., \& Wiltbank, J. N. (1982). Effect of steroids and/or 48 HR calf removal on serum luteinizing hormone concentrations in anestrous beef cows. Theriogenology, 18(3), 349-356. https://doi.org/10.1016/0093-691X(82)90012-7

Williams, G. L. (1990). Suckling as a regulator of postpartum rebreeding in cattle: a review. Journal of Animal Science, 68(3), 831. https://doi.org/10.2527/1990.683831x

Wiltbank, M. C., Gümen, A., \& Sartori, R. (2002). Physiological classification of anovulatory conditions in cattle. Theriogenology, 57(1), 21-52.

Yavas, Y., \& Walton, J. S. (2000). Postpartum acyclicity in suckled beef cows: A review. Theriogenology, 54(1), 25-55. https://doi.org/10.1016/S0093-691X(00)00323-X

Recebido: 9 de jul

Aprovado: 30 de junho, 2020.

Disponível online: 23 de outubro, 2020.
Licenciamento: Este artigo é publicado na modalidade Acesso Aberto sob a licença Creative Commons Atribuição 4.0 (CC-BY 4.0), a qual permite uso irrestrito, distribuição, reprodução em qualquer meio, desde que o autor e a fonte sejam devidamente creditados. 